\title{
Tutorial: How to Value Software in a Business, and Where Might the Value Go?
}

\author{
Gio Wiederhold \\ Professor Emeritus \\ Stanford University and MITRE Corporation \\ Stanford CA 94305-9040 \\ USA
}

This tutorial consists of two parts. In the first part we motivate why businesses should determine the value of the Intellectual Property (IP) inherent in software and present a method to determine that value in a simple business model: Enterprise software for sale. In the second part we consider the same software as marketed in a service model. A service model imposes a maintenance obligation. Maintenance is often outsourced so we also consider the risk to IP, especially when work is performed offshore. Finally we present the consequences to the original creators when IP is segregated into a tax haven.

There exists a voluminous literature on estimation of the cost of producing software, but that literature largely ignores the benefits of using that software. While software creators believe that their products are valuable, they are rarely called upon to quantify its benefits. Evaluation of software and its benefits in commerce is left to lawyers, economists, software vendors, or promoters. The results are often inconsistent. For modern enterprises intellectual capital greatly exceeds the tangible capital on the books, but is not shown in their annual reports. The problem is that the value of software is essentially independent of the cost and effort spent to create it. A few brilliant lines of code can have a very high value, whereas a million lines of code that generate a report that nobody uses have little value. The presentation will present a method for valuing software based on the income that sales of a software product are expected to generate, following accepted economic principles. An issue specific to software is that software is continually being upgraded, and is hence more squishy than say books or music. The steps of the valuation process are integrated via a simple quantitative example.

Having a quantitative model on a spreadsheet allows exploration of alternatives. That capability is shown by evaluating also a service business model alternative. In that setting we can also determine why software has a finite life, although it does not wear out as tangibles do. The model is being used now for international IP transfer pricing. When companies outsource part of their operations they must also give access to the required IP. If that IP is not properly valued distortions occur in decision-making processes, and risks cannot be quantified. These distortions can have very large effects on the income and capital accumulation in the home and destination country. If a taxhaven is interposed as a holder of the IP the consequences for creators, the stockholders, and their countries can be amazing, and are rarely understood by the participants.

P. van Eck, J. Gordijn, and R. Wieringa (Eds.): CAiSE 2009, LNCS 5565, pp. 9-10, 2009

(C) Springer-Verlag Berlin Heidelberg 2009 
Awareness of the value of the product of a creator's knowledge and effort can help in making decisions on the design and the implementation focus. Some further conclusions are drawn from the modeling results that reflect on academic and business practice. A paper on the method used has appeared in the Comm. of the ACM, September 2006, but could not cover all of the issues. Links to further material are available at http://infolab.stanford.edu/pub/gio/ inprogress.html\#worth. 